\title{
Association between the non-HDL- cholesterol to HDL-cholesterol ratio and non-alcoholic fatty liver disease in Chinese children and adolescents: a large single- center cross-sectional study
}

Shouxing Yang ${ }^{1} \mathbb{D}$, Jinwei Zhong ${ }^{1}$, Mengsi Ye ${ }^{1}$, Lei Miao ${ }^{1}$, Guangrong $\mathrm{Lu}^{1}$, Changlong Xu${ }^{1}$, Zhanxiong Xue ${ }^{1 *}$ and Xinhe Zhou ${ }^{2^{*}}$

\begin{abstract}
Background: The non-HDL-cholesterol to HDL-cholesterol (NHDLC/HDLC) ratio is closely related to a variety of dyslipidemia-related diseases. This study examined the relationship between the NHDLC/HDLC ratio and nonalcoholic fatty liver (NAFLD) in children and adolescents.

Methods: This cross-sectional survey included a total of 7759 eligible Chinese children and adolescents (5692 boys and 2067 girls) who received routine medical examinations. The anthropometric and laboratory data of the subjects were collected. NAFLD was diagnosed by liver ultrasonography. Binary logistic regression analysis was performed on the NHDLC/HDLC ratio, NHDLC, HDLC and NAFLD. Receiver operating characteristic (ROC) curve analysis was used to compare the diagnostic significance of the above parameters for NAFLD.
\end{abstract}

Results: The total prevalence of NAFLD was 4.36\%, and the prevalence in boys was higher than that in girls (5.61\% vs. $1.9 \%, P<0.001)$. The prevalence of NAFLD was positively correlated with the NHDLC/HDLC ratio $(P<0.001)$. The binary logistic regression analysis demonstrated that the OR was $8.61(95 \% \mathrm{Cl}, 5.90-12.57, P<0.001)$ in tertile 3 (highest NHDLC/HDLC ratio) compared with tertile 1 (lowest NHDLC/HDLC ratio). After adjustment for age, sex, body mass index (BMI), alanine aminotransferase (ALT), uric acid (UA), total bilirubin (TB), fasting plasma glucose (FPG) and Homeostasis Model Assessment of Insulin Resistance (HOMA-IR), the OR for tertile $3(\mathrm{OR}=1.83,95 \% \mathrm{Cl}$, 1.04-3.22, $P=0.035$ ) was still significantly higher than that of tertile 1 . The area under the curve (AUC) of the NHDL C/HDLC ratio of boys was 0.787 , which was significantly greater than NHDLC and HDLC $(0.719$ and $0.726, P<0.001)$. For girls, the AUC of the NHDLC/HDLC ratio was 0.763 , which was also significantly greater than NHDLC $(0.661, P<$ (Continued on next page)

\footnotetext{
*Correspondence: 1481013118@qq.com; 2206251@qq.com

'Department of Gastroenterology, The Second Affiliated Hospital and Yuying

Children's Hospital of Wenzhou Medical University, Wenzhou 325000,

Zhejiang, China

${ }^{2}$ Department of Endocrinology, The Second Affiliated Hospital and Yuying Children's Hospital of Wenzhou Medical University, Wenzhou 325000,

Zhejiang, China
}

(c) The Author(s). 2020 Open Access This article is licensed under a Creative Commons Attribution 4.0 International License, which permits use, sharing, adaptation, distribution and reproduction in any medium or format, as long as you give appropriate credit to the original author(s) and the source, provide a link to the Creative Commons licence, and indicate if changes were made. The images or other third party material in this article are included in the article's Creative Commons licence, unless indicated otherwise in a credit line to the material. If material is not included in the article's Creative Commons licence and your intended use is not permitted by statutory regulation or exceeds the permitted use, you will need to obtain permission directly from the copyright holder. To view a copy of this licence, visit http://creativecommons.org/licenses/by/4.0/. The Creative Commons Public Domain Dedication waiver (http://creativecommons.org/publicdomain/zero/1.0/) applies to the data made available in this article, unless otherwise stated in a credit line to the data. 
(Continued from previous page)

0.001). The cutoff point of the NHDLC/HDLC ratio was 2.475 in boys and 2.695 in girls. In addition, the AUC of the NHDLC/HDLC ratio was 0.761 in subjects with normal ALT levels (ALT $\leq 40 \mathrm{U} / \mathrm{L}$ ), which was significantly higher than $\operatorname{NHDLC}(0.680, P<0.001)$ and HDLC $(0.724, P=0.007)$. For subjects with elevated ALT levels $(A L T>40 \cup / L)$, the AUC of the NHDLC/HDLC ratio (0.746) was also significantly greater than $\mathrm{NHDLC}(0.646, P<0.001)$.

Conclusions: The NHDLC/HDLC ratio was positively correlated with NAFLD in Chinese children and adolescents. It may serve as an effective indicator to help identify NAFLD in children and adolescents.

Keywords: Non-alcoholic fatty liver disease, NHDLC/HDLC ratio, Children, Adolescents, Cross-sectional study, Receiver operating characteristic curve

\section{Background}

Non-alcoholic fatty liver disease (NAFLD) is a chronic liver disease (CLD) involving fatty liver, liver steatosis and nonalcoholic steatohepatitis (NASH) [1]. Currently, NAFLD is the major reason for CLD in the pediatric population. Given the epidemiological data, the prevalence of NAFLD is $2.6-7.1 \%$ of all children and approximately $27.8-41.2 \%$ of obese children. Furthermore, the incidence ratio of NAFLD is on the rise $[2,3]$.

NAFLD is recognized as metabolic syndrome (MS) that manifested in the hepatic area, and its progressive form NASH increases the risk of liver cancer, end-stage liver disease and cirrhosis [4]. Pediatric NAFLD is considered to be involved in the pathogenesis of diabetes mellitus (DM), MS and cardiovascular disease (CVD) [5]. Since children with NAFLD are usually asymptomatic, the diagnosis of NAFLD in childhood and adolescence is challenging. Although the diagnostic gold standard for NAFLD is liver biopsy, it is not readily accepted by children because of its invasive nature. Liver ultrasonography is a feasible and noninvasive means of diagnosing NAFLD, but not all children undergo liver ultrasonography because their parents may be reluctant to let them receive medical examination for a clinically silent disease [6]. Therefore, valuable indicators for NAFLD should be identified for early detection and prevention of later progression.

The non-HDL-cholesterol to HDL-cholesterol (NHDL C/HDLC) ratio is closely related to a variety of dyslipidemia-related diseases, such as chronic kidney disease, gallbladder polyp, insulin resistance (IR) and MS. In addition, the NHDLC/HDLC ratio has a better predictive value for CVD in type $2 \mathrm{DM}$ than high-density lipoprotein cholesterol (HDLC) [7-10]. A recent prospective study found that the NHDLC/HDLC ratio is more sensitive for predicting NAFLD in adults than non-high-density lipoprotein cholesterol (NHDLC) [11]. However, the association between the NHDLC/HDLC ratio and NAFLD in childhood and adolescence remains unclear. Therefore, This study examined the relationship between the NHDLC/HDLC ratio and NAFLD in childhood and adolescence.

\section{Materials and methods Subjects}

A total of 7759 children and adolescents who received regular medical examinations from January 2015 to January 2020 in the Second Affiliated Hospital of Wenzhou Medical University were investigated. The inclusion criteria were subjects aged 2-18 years who had liver ultrasonography. Patients with hepatitis virus infection, metabolic liver disease, lipid-lowering agent treatment in the last month, hereditary hyperlipidemia or regular alcohol consumption were excluded.

\section{The elements of anthropometry and laboratory examinations}

The clinical and anthropometric measurements assessed were age, sex, weight and height. Height and weight were examined on the same day. Body weight $(\mathrm{kg}) \mathrm{di}-$ vided by height squared $\left(\mathrm{m}^{2}\right)$ was used to calculate the body mass index (BMI). This study used fasting blood samples to assess metabolic variables using standard laboratory testing. Data collected for this study included total cholesterol (TC), fasting plasma glucose (FPG), HDLC, triglycerides (TG), alanine aminotransferase (ALT), alkaline phosphatase (APL), glycated hemoglobin A1c (HbA1c), low-density lipoprotein cholesterol (LDLC), albumin (ALB), uric acid (UA), aspartate aminotransferase (AST), gamma-glutamyl transferase (GGT), total bilirubin (TB) and creatinine ( $\mathrm{Cr}$ ). Homeostasis Model Assessment of IR (HOMA-IR) was calculated by $\mathrm{HOMA}-\mathrm{IR}=\mathrm{FPG} \times$ fasting insulin/22.5 [12]. Children with $\mathrm{BMI}>95$ th percentile were classified as obese [13].

\section{Ultrasonography}

Liver ultrasonography was performed by an experienced expert with a Philips En2visor26 type ultrasonic diagnostic instrument (probe frequency 3.5-5.0 M Hz). The diagnosis of NAFLD was made according to the following ultrasonic criteria: ultrasound signals suggesting deep attenuation, vascular blurring, and discursively expanded echo (bright) liver showing echoes on the kidney or spleen [14]. 


\section{Statistical analysis}

All data were evaluated using SPSS 21.0 (SPSS Inc., Chicago, IL, USA) and EmpowerStats (http://www.empowerstats.com). Means \pm standard deviations (SD) denoted continuous data. Categorical variables were described using percentages. All participants were stratified into three tertiles according to the NHDLC/HDLC ratio $(\leq 1.79,1.80-2.36, \geq 2.37)$. To evaluate the differences between groups, this study performed one-way ANOVA followed by the least significant difference (LSD) test on continuous variables with Gaussian distribution. The chi squared $\left(x^{2}\right)$ test was performed on the categorical data. Binary logistic regression analysis was performed to evaluate the association between the NHDLC/HDLC ratio and NAFLD. The NHDLC/HDLC ratio was calculated as medians with interquartile ranges (IQR; IQR = Q3-Q1, where Q3 is the third quartile and Q1 is the first quartile of data distribution). The potential confounders used for adjustment included age, sex, BMI, ALT, UA, TB, FPG and HOMA-IR. Receiver operating characteristic (ROC) curve analysis was performed to evaluate the effectiveness of different lipid levels as indicators for NAFLD. The $P$ value for a two-tailed test < 0.05 reflected statistical significance, and the figures were generated by GraphPad Prism 6 (GraphPad Software Inc., San Diego, CA, USA).

\section{Results}

\section{Baseline characteristics}

As shown in Fig. 1, a total of 7759 eligible children and adolescents (5692 boys, 2067 girls) were recruited. The mean age of the participants was $9.09 \pm 3.30$ years, and the mean BMI was $17.38 \pm 3.72 \mathrm{~kg} / \mathrm{m}^{2}$. Table 1 compares the baseline characteristics by tertiles of the NHDLC/ HDLC ratio. These three tertiles were significantly different. In contrast to subjects in tertile 3 (highest NHDL
C/HDLC ratio), subjects in tertile 1 (lowest NHDLC/ HDLC ratio) had lower BMI, ALT, GGT, UA, ALB, TC, TG, LDLC, HbA1c (\%), FPG and HOMA-IR and higher TB, ALP and HDLC $(P<0.001)$. The incidence of obesity noticeably increased across NHDLC/HDLC tertiles 1,2 and 3 (4.81, 9.22 and $21.18 \%$, respectively, $P<0.001$ ).

\section{Prevalence of NAFLD and the NHDLC/HDLC ratio}

The prevalence of NAFLD was positively correlated with the NHDLC/HDLC ratio. Tertile $2(2.33 \%, P<0.001)$ and tertile $3(9.49 \%, P<0.001)$ showed a significantly higher NAFLD prevalence compared with tertile 1 $(1.2 \%)$. The prevalence of NAFLD in tertile 3 was nearly eight times higher than tertile 1 (Fig. 2). In addition, the total prevalence of NAFLD was $4.36 \%$, and the prevalence for boys was higher than girls $(5.61 \%$ vs. $1.9 \%, P<$ 0.001) (Fig. 3).

\section{Association between the NHDLC/HDLC ratio and NAFLD}

The binary logistic regression analysis showed that the odds ratio (OR) of NAFLD increased significantly with the increase in the NHDLC/HDLC ratio. The OR was 8.61 (95\% CI, 5.90-12.57, $P<0.001$ ) in tertile 3 compared with tertile 1 . When potential confounders (age, sex, BMI, ALT, UA, TB, FPG and HOMA-IR) were adjusted, the OR for tertile $3(\mathrm{OR}=1.83,95 \% \mathrm{CI}, 1.04-$ $3.22, P=0.035)$ was still significantly higher than tertile 1. Moreover, with the per IQR increase in the NHDLC/ HDLC ratio, the OR for NAFLD was 1.89 (95\% CI, 1.52-2.34, $P<0.001$ ). (Table 2).

\section{The diagnostic significance of the NHDLC/HDLC ratio for NAFLD}

To assess the diagnostic significance of the NHDLC/ HDLC ratio, NHDLC and HDLC for NAFLD, this study used sex-specific ROC curve analysis (Fig. 4). The area

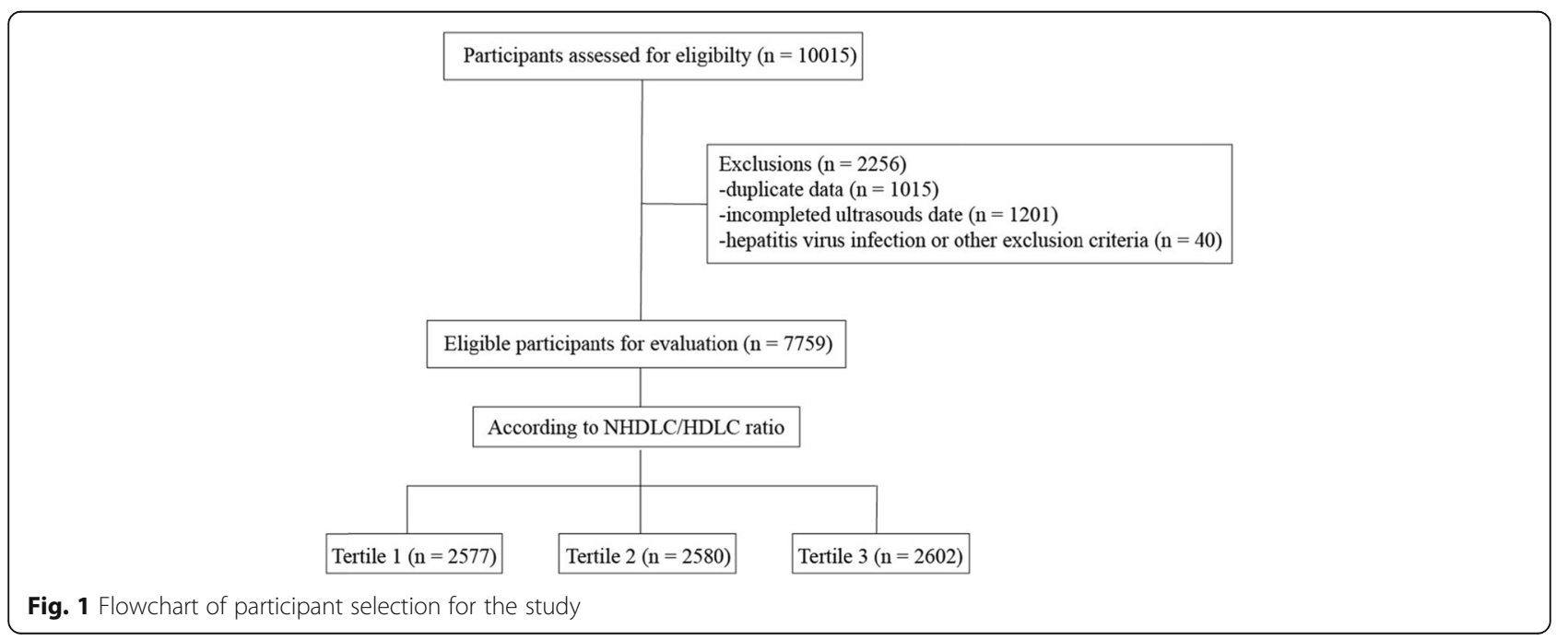


Table 1 Baseline characteristics of participants

\begin{tabular}{|c|c|c|c|c|}
\hline \multirow[t]{3}{*}{ Characteristics } & \multicolumn{3}{|c|}{ NHDLC/HDLC ratio } & \multirow{3}{*}{$\begin{array}{l}P \\
\text { value }\end{array}$} \\
\hline & Tertile 1 & Tertile 2 & Tertile 3 & \\
\hline & $(\leq 1.79)$ & $(1.80-2.36)$ & $(\geq 2.37)$ & \\
\hline Sample size & 2577 & 2580 & 2602 & \\
\hline Age (year) & $9.24 \pm 3.11$ & $9.00 \pm 3.31^{b}$ & $9.03 \pm 3.47$ & 0.017 \\
\hline Sex, n (\%) & & & & $<0.001$ \\
\hline Girl & 771 (29.92) & $950(36.82)^{b}$ & $906(34.82)^{a}$ & \\
\hline Boy & $1806(70.08)$ & $1630(63.18)^{b}$ & $1696(65.18)^{a}$ & \\
\hline $\mathrm{BMI}\left(\mathrm{kg} / \mathrm{m}^{2}\right)$ & $16.47 \pm 2.64$ & $17.02 \pm 3.27^{b}$ & $18.64 \pm 4.60^{\mathrm{a} / \mathrm{c}}$ & $<0.001$ \\
\hline $\mathrm{ALT}(\mathrm{U} / \mathrm{L})$ & $15.12 \pm 8.49$ & $15.93 \pm 17.55$ & $19.27 \pm 19.88^{\mathrm{a} / \mathrm{c}}$ & $<0.001$ \\
\hline AST (U/L) & $26.51 \pm 6.75$ & $26.64 \pm 13.29$ & $27.09 \pm 13.62$ & 0.175 \\
\hline $\mathrm{ALP}(\mathrm{U} / \mathrm{L})$ & $252.60 \pm 78.46$ & $242.84 \pm 71.71^{b}$ & $240.50 \pm 76.05^{a}$ & $<0.001$ \\
\hline GGT (U/L) & $12.29 \pm 5.27$ & $12.83 \pm 6.02^{b}$ & $15.45 \pm 9.98^{\mathrm{a} / \mathrm{c}}$ & $<0.001$ \\
\hline $\mathrm{TB}(\mathrm{mmol} / \mathrm{L})$ & $9.88 \pm 4.38$ & $9.66 \pm 4.36$ & $9.27 \pm 4.14^{\mathrm{ac}}$ & $<0.001$ \\
\hline $\mathrm{Cr}(\mu \mathrm{mol} / \mathrm{L})$ & $39.50 \pm 8.75$ & $39.31 \pm 9.48$ & $39.35 \pm 10.24$ & 0.749 \\
\hline UA $(\mu \mathrm{mol} / \mathrm{L})$ & $300.55 \pm 69.92$ & $305.06 \pm 73.84$ & $327.42 \pm 85.44^{\mathrm{a} / \mathrm{c}}$ & $<0.001$ \\
\hline ALB (g/L) & $45.36 \pm 2.22$ & $45.48 \pm 2.17$ & $45.78 \pm 2.33^{\mathrm{a} / \mathrm{c}}$ & $<0.001$ \\
\hline $\mathrm{TC}(\mathrm{mmol} / \mathrm{L})$ & $4.09 \pm 0.63$ & $4.27 \pm 0.65^{b}$ & $4.62 \pm 0.82^{a / c}$ & $<0.001$ \\
\hline $\mathrm{TG}(\mathrm{mmol} / \mathrm{L})$ & $0.77 \pm 0.30$ & $0.89 \pm 0.36^{b}$ & $1.21 \pm 0.64^{\mathrm{a} / \mathrm{c}}$ & $<0.001$ \\
\hline $\mathrm{HDLC}(\mathrm{mmol} / \mathrm{L})$ & $1.67 \pm 0.29$ & $1.39 \pm 0.22^{b}$ & $1.17 \pm 0.21^{a / c}$ & $<0.001$ \\
\hline LDLC (mmol/L) & $1.90 \pm 0.45$ & $2.31 \pm 0.46^{b}$ & $2.79 \pm 0.67^{\mathrm{a} / \mathrm{c}}$ & $<0.001$ \\
\hline $\mathrm{HbA1c}(\%)$ & $5.43 \pm 0.29$ & $5.43 \pm 0.27$ & $5.46 \pm 0.29^{a / c}$ & 0.001 \\
\hline FPG (mmol/L) & $4.68 \pm 0.39$ & $4.70 \pm 0.38$ & $4.73 \pm 0.37^{\mathrm{a} / c}$ & $<0.001$ \\
\hline HOMA-IR & $1.42 \pm 1.28$ & $1.67 \pm 1.93^{b}$ & $2.16 \pm 2.07^{\mathrm{a} / \mathrm{c}}$ & $<0.001$ \\
\hline Obesity [n (\%)] & $124(4.81 \%)$ & $238(9.22 \%)^{b}$ & $551(21.18 \%)^{a / c}$ & $<0.001$ \\
\hline
\end{tabular}

Data are expressed as the mean \pm SD or percentage

$B M I$ Body mass index, ALT Alanine aminotransferase, AST Aminotransferase, ALP Alkaline phosphatase, GGT Gamma-glutamyl transferase, TB Total bilirubin, Cr Creatinine, UA Uric acid, ALB Albumin, TC Total cholesterol, TG Triglycerides, HDLC High-density lipoprotein cholesterol, LDLC Low-density lipoprotein cholesterol, HbA1c Glycated hemoglobin A1c, FPG Fasting plasma glucose, HOMA-IR Homeostasis Model Assessment of Insulin Resistance

${ }^{\text {a }} P<0.05$, tertile 3 compared with tertile 1

${ }^{\mathrm{b}} \mathrm{P}<0.05$, tertile 2 compared with tertile 1

${ }^{\mathrm{c}} P<0.05$, tertile 3 compared with tertile 2
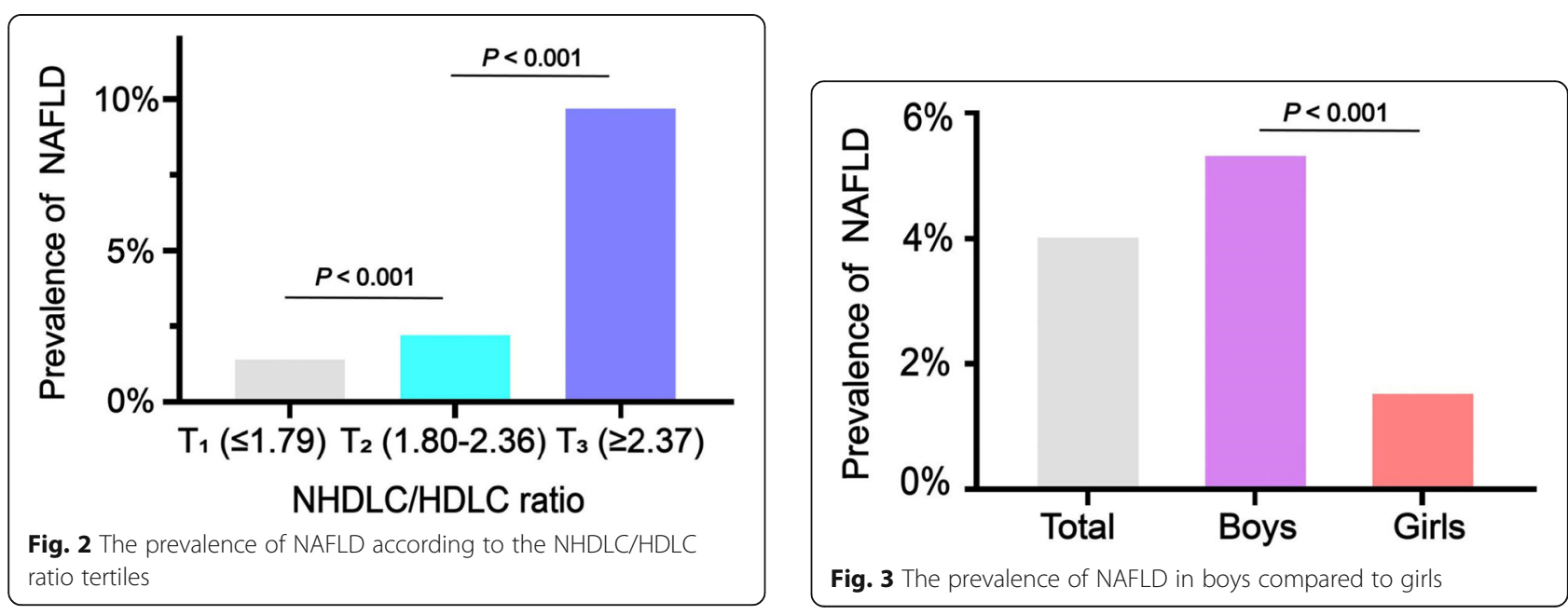
Table 2 ORs for NAFLD by NHDLC/HDLC ratio

\begin{tabular}{|c|c|c|c|c|c|c|}
\hline \multirow{2}{*}{$\begin{array}{l}\text { NHDLC/HDLC } \\
\text { ratio }\end{array}$} & \multicolumn{3}{|c|}{ Crude } & \multicolumn{3}{|c|}{ Adjusted $^{\mathrm{a}}$} \\
\hline & $\bar{\beta}$ & OR $(95 \% \mathrm{Cl})$ & $P$ & $\bar{\beta}$ & OR $(95 \% \mathrm{Cl})$ & $P$ \\
\hline Tertile $1(\leq 1.79)$ & & 1 & & & 1 & \\
\hline Tertile 2 (1.80-2.36) & 0.67 & $1.96(1.16-3.03)$ & 0.002 & 0.07 & $1.07(0.57-2.04)$ & 0.824 \\
\hline Tertile 3 ( $\geq 2.37$ ) & 2.15 & $8.61(5.90-12.57)$ & 0.000 & 0.61 & $1.83(1.04-3.22)$ & 0.035 \\
\hline Per IQR (=0.9) & 1.06 & $2.88(2.58-3.22)$ & 0.000 & 0.63 & $1.89(1.52-2.34)$ & 0.000 \\
\hline
\end{tabular}

Data are coefficient $(\beta)$, odds ratio (OR) and $95 \%$ confidence intervals $(\mathrm{Cl}), P$ value

NAFLD Non-alcoholic fatty liver disease, NHDLC/HDLC Non-HDL-cholesterol to HDL-cholesterol, IQR Interquartile range

Adjusted $^{\text {a }}$ for age, sex, BMI, ALT, UA, TB, FPG and HOMA-IR

under the curve (AUC) of the NHDLC/HDLC ratio was $0.787(0.758-0.816)$ in boys, which was significantly greater than NHDLC $(0.719,0.687-0.751, P<0.001)$ and HDLC $(0.726,0.698-0.755, P<0.001)$. For girls, the AUC of the NHDLC/HDLC ratio (0.763, 0.688-0.837) was also significantly greater than NHDLC (0.661, $0.580-0.743, P<0.001$ ), but there was no considerable difference from HDLC (0.732, 0.663-0.802, $P=0.239$ ). The cutoff point of the NHDLC/HDLC ratio in boys was 2.475 , exhibiting a sensitivity of $71.18 \%$ and a specificity of $74.46 \%$. The cutoff point in girls was 2.695 , exhibiting a sensitivity of $66 \%$ and a specificity of $80.09 \%$. Besides, Fig. 5 shows the ROC curves for the different levels of ALT. The AUC of the NHDLC/HDLC ratio was $0.761(0.725-0.798)$ in subjects with normal ALT levels (ALT $\leq 40 \mathrm{U} / \mathrm{L}$ ), which was significantly greater than NHDLC $(0.680,0.642-0.718, P<0.001)$ and HDLC $(0.724,0.689-0.758, P=0.007)$. For subjects with elevated ALT levels (ALT >40 U/L), the AUC of the NHDLC/HDLC ratio $(0.746,0.687-0.806)$ was also significantly greater than NHDLC $(0.646,0.579-0.713, P<$ 0.001 ), but there was no considerable difference with $\operatorname{HDLC}(0.715,0.652-0.778, P=0.226)$.

\section{Discussion}

This study is the first to find that a high NHDLC/HDLC ratio was highly positively correlated with the prevalence of NAFLD in childhood and adolescence. Moreover, the diagnostic value of the NHDLC/HDLC ratio for NAFLD was better than NHDLC in both boys and girls. Therefore, the NHDLC/HDLC ratio may assist in the early identification of NAFLD in childhood and adolescence.

Previous studies confirmed that MS and obesity were the major risk elements for NAFLD in the pediatric population and dyslipidemia played a pivotal role in NAFLD pathogenesis $[4,15]$. Recent clinical assessments suggested that statin-based therapies (lipid-lowering agent treatment) improved impaired hepatic function in patients with NAFLD [16]. The dyslipidemia in NAFLD is symbolized by increased levels of TG and decreased levels of HDLC [17]. Driven by lipid metabolism abnormalities, excess fat in NAFLD accumulates in hepatocytes. The mentioned intrahepatic lipid accumulation was attributed to decreased TG export, uptake of liver free fatty acids (FFAs) and very low-density lipoprotein cholesterol production $[18,19]$. Ectopic lipid overloading in hepatocytes is associated with inflammation induction, oxidative stress, and the secretion of numerous cytokines (including adiponectin, interleukins and tumor necrosis factor) [20]. Moreover, excess FFAs form fatty acyl-CoAs sequentially catalyzed by fatty acyl-CoA synthetase, which likely induces $\beta$-oxidation pathways. The above inflammation and oxidative stress are involved in NAFLD initiation and progression [21, 22].

NHDLC, which refers to TC minus HDLC, is regarded as a secondary target of the lipid-lowering therapeutic method [23]. Compared to NHDLC, the NHDLC/HDLC ratio covers more comprehensive abilities of lipid dysregulation and a better capacity to assess lipid metabolism-related disease risk. According to the existing study, the NHDLC/HDLC ratio was better than NHDLC in predicting the occurrence of CVD in type 2 DM [10], and the ratio exhibited a higher prediction result than the apoB/apoA1 ratio of IR and MS [9]. A prospective cohort study revealed that the NHDLC/HDLC ratio outperformed NHDLC in predicting new-onset NAFLD in the Chinese adult population [11]. Therefore, it is necessary to examine the association between the NHDLC/HDLC ratio and NAFLD in the pediatric population.

Baseline characteristic data of 7759 subjects were stratified into three tertiles according to the NHDLC/ HDLC ratio, and the results showed that laboratory variables (such as ALT, TG, LDLC, HbA1c and FPG) were higher in the highest NHDLC/HDLC ratio tertile than the lowest tertile. Similar results were reported in a previous study [24]. As expected, the incidence of obesity notably increased across NHDLC/HDLC tertiles. The prevalence of NAFLD increased with increasing tertiles (NHDLC/HDLC ratio). The binary logistic regression analysis suggested that a higher NHDLC/HDLC ratio was positively correlated to NAFLD. In terms of sex distribution, the frequency of NAFLD by sex was significantly higher in boys than girls. The results of this study complies with Brunt et al. [25] and Welsh et al. [26]. This 

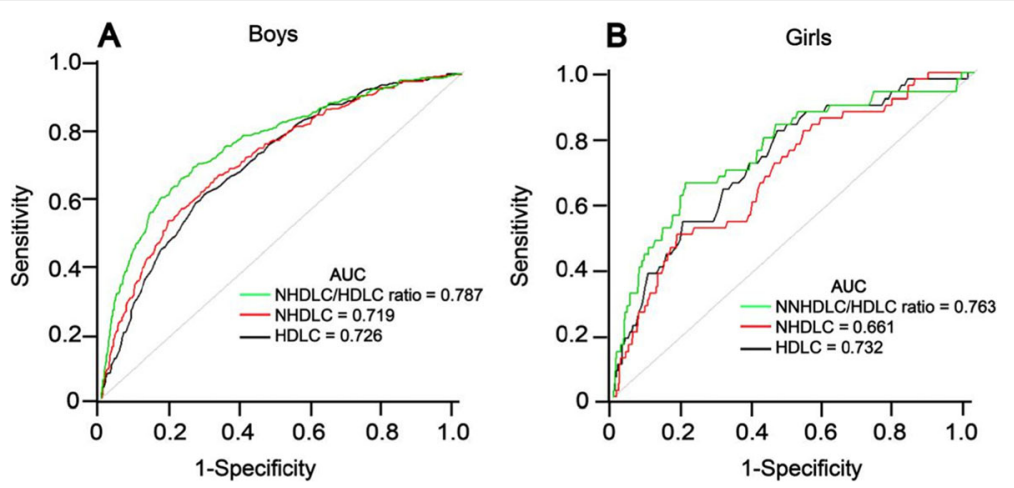

Fig. 4 ROC curves of NHDLC/HDLC ratio, HDLC and NHDLC for NAFLD in boys (a) and girls (b)

phenomenon may be attributed to the potential protective role of estrogen against hepatic steatosis [27, 28].

The findings here have certain clinical implications. The AUC results suggest that the NHDLC/HDLC ratio may be exercised as a more suitable indicator for NAFLD in childhood and adolescence than NHDLC and HDLC, especially in boys. Moreover, this study also assessed the diagnostic value of the NHDLC/HDLC ratio for NAFLD at different levels of ALT, and the results showed that the NHDLC/ HDLC ratio was useful even in subjects with normal ALT levels. In addition, the NHDLC/HDLC ratio exhibits simplicity and feasibility for determination. Therefore, the NHDLC/HDLC ratio is feasible for screening and identifying NAFLD in children and adolescents.

\section{Study strengths and limitations}

The conclusions of this large sample study are more convincing and meaningful. It was the first study to find that the NHDLC/HDLC ratio was positively associated with NAFLD in children and adolescents in China. The NHDLC/HDLC ratio exhibits simplicity and feasibility for determination, accordingly, the NHDLC/HDLC ratio may be helpful for identifying NAFLD in children and adolescents.
Some limitations are worth noting here. First, the NAFL $\mathrm{D}$ diagnosis was performed using ultrasound instead of liver biopsy to avoid the complications and invasiveness of liver biopsy. However, the NAFLD sensitivity of ultrasonography is reduced when the body fat percentage is $<30 \%$, which may lead to misdiagnosis [29]. Second, since this was a single-center study, multicenter studies should be performed to confirm the conclusions. Third, this study was a cross-sectional study, therefore, the causal relationship between the NHDLC/HDLC ratio and NAFLD cannot be determined as a whole. This correlation needs further confirmation form prospective study. Fourth, since this study was performed only in Chinese children and adolescents, the association between the NHDLC/HDLC ratio and NAFLD needs to be confirmed in populations from other regions and ethnicities.

\section{Conclusions}

The NHDLC/HDLC ratio is positively correlated with NAFLD in Chinese children and adolescents. It may be an effective indicator to help identify NAFLD in childhood and adolescence and prevent disease progression.
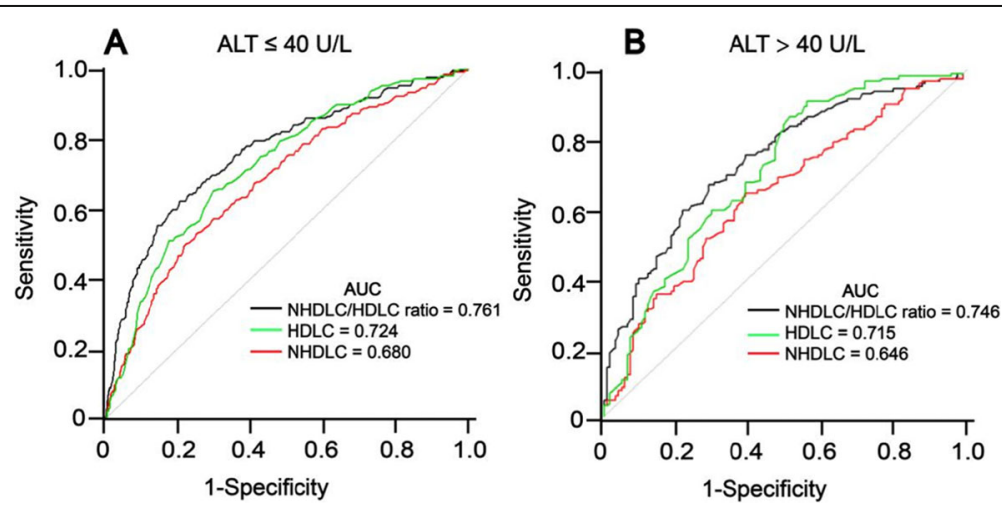

Fig. 5 ROC curves of NHDLC/HDLC ratio, HDLC and NHDLC for NAFLD in subjects with normal ALT levels (a) and elevated ALT levels (b) 


\section{Abbreviations}

NHDLC/HDLC: Non-HDL-cholesterol to HDL-cholesterol; NAFLD: Nonalcoholic fatty liver disease; CLD: Chronic liver disease; NASH: Nonalcoholic steatohepatitis; MS: Metabolic syndrome; DM: Diabetes mellitus; CVD: Cardiovascular disease; IR: Insulin resistance; HDLC: High-density lipoprotein cholesterol; NHDLC: Non-high-density lipoprotein cholesterol; BMI: Body mass index; TC: Total cholesterol; FPG: Fasting plasma glucose; TG: Triglycerides; ALT: Alanine aminotransferase; ALP: Alkaline phosphatase; HbA1c: Glycated hemoglobin A1c; LDLC: Low-density lipoprotein cholesterol; ALB: Albumin; UA: Uric acid; AST: Aminotransferase; GGT: Gamma-glutamyl transferase; TB: Total bilirubin; Cr: Creatinine; HOMA-IR: Homeostasis Model Assessment of Insulin Resistance; OR: Odds ratio; IQR: Interquartile ranges; Cl: Confidence intervals; ROC: Receiver operating characteristic; AUC: Area under the curve; FFAs: Free fatty acids

\section{Acknowledgements}

Great thanks to Xinhe Zhou (Department of Endocrinology, The Second Affiliated Hospital and Yuying Children's Hospital of Wenzhou Medical University) for her helpful comments on the study.

\section{Authors' contributions}

SXY and XHZ designed the study and wrote the manuscript. MSY, GRL, LM and JWZ collected anthropometric measurements and laboratory tests data. CLX and XZX analyzed the data. All authors approved the final manuscript.

\section{Funding}

The project was supported by the Medical Health Science and Technology Project of Zhejiang Provincial Health Commission (No. 2019330727).

\section{Availability of data and materials}

All data used in this study are available from the corresponding author.

\section{Ethics approval and consent to participate}

This cross-sectional study protocol obtained approval from the Ethics Committee of the Second Affiliated Hospital of Wenzhou Medical University (No. L-2020-21). All children's parents provided informed consent.

\section{Consent for publication}

Not applicable.

\section{Competing interests}

The authors declare that they have no competing interests.

Received: 20 August 2020 Accepted: 17 November 2020

Published online: 22 November 2020

\section{References}

1. Lomonaco R, Bril F, Portillo-Sanchez P, Ortiz-Lopez C, Orsak B, Biernacki D, et al. Metabolic impact of nonalcoholic Steatohepatitis in obese patients with type 2 diabetes. Diabetes Care. 2016;39:632-8.

2. Alisi A, Feldstein AE, Villani A, Raponi M, Nobili V. Pediatric nonalcoholic fatty liver disease: a multidisciplinary approach. Nat Rev Gastroenterol Hepatol. 2012:9:152-61.

3. Anderson EL, Howe LD, Jones HE, Higgins JP, Lawlor DA, Fraser A. The prevalence of non-alcoholic fatty liver disease in children and adolescents: a systematic review and meta-analysis. PLoS One. 2015;10:e0140908.

4. Katsiki N, Mikhailidis DP, Mantzoros CS. Non-alcoholic fatty liver disease and dyslipidemia: an update. Metab Clin Exp. 2016;65:1109-23.

5. Nobili V, Alkhouri N, Alisi A, Della Corte C, Fitzpatrick E, Raponi M, et al. Nonalcoholic fatty liver disease: a challenge for pediatricians. JAMA Pediatr. 2015;169:170-6.

6. Kim JY, Cho J, Yang HR. Biochemical predictors of early onset non-alcoholic fatty liver disease in young children with obesity. J Korean Med Sci. 2018;33: e122.

7. Zuo PY, Chen XL, Liu YW, Zhang R, He XX, Liu CY. Non-HDL-cholesterol to $\mathrm{HDL}$-cholesterol ratio as an independent risk factor for the development of chronic kidney disease. Nutr Metab Cardiovasc Dis. 2015;25:582-7.

8. Zhao X, Zheng $H$, Shan S, Wang K, Zhang M, Xie S, et al. Association between the non-HDL-cholesterol-to-HDL-cholesterol ratio and the risk of gallbladder polyp formation among men: a retrospective cohort study. Lipids Health Dis. 2020;19:146.
9. Kim SW, Jee JH, Kim HJ, Jin SM, Suh S, Bae JC, et al. Non-HDL-cholesterol/ $\mathrm{HDL}$-cholesterol is a better predictor of metabolic syndrome and insulin resistance than apolipoprotein B/apolipoprotein A1. Int J Cardiol. 2013;168: 2678-83.

10. Lu W, Resnick HE, Jablonski KA, Jones KL, Jain AK, Howard WJ, et al. NonHDL cholesterol as a predictor of cardiovascular disease in type 2 diabetes: the strong heart study. Diabetes Care. 2003;26:16-23.

11. Wang K, Shan S, Zheng H, Zhao X, Chen C, Liu C. Non-HDL-cholesterol to $\mathrm{HDL}$-cholesterol ratio is a better predictor of new-onset non-alcoholic fatty liver disease than non-HDL-cholesterol: a cohort study. Lipids Health Dis. 2018;17:196.

12. Hu Y, Li L, Xu Y, Yu T, Tong G, Huang H, et al. Short-term intensive therapy in newly diagnosed type 2 diabetes partially restores both insulin sensitivity and $\beta$-cell function in subjects with long-term remission. Diabetes Care. 2011:34:1848-53.

13. Hill AP, Zuckerman KE, Fombonne E. Obesity and autism. Pediatrics. 2015; 136:1051-61.

14. Farrell GC, Chitturi S, Lau GK, Sollano JD. Asia-Pacific working party on NAFL D: guidelines for the assessment and management of non-alcoholic fatty liver disease in the Asia-Pacific region: executive summary. J Gastroenterol Hepatol. 2007;22:775-7.

15. Fang $Y L$, Chen $H$, Wang $C L$, Liang $L$. Pathogenesis of non-alcoholic fatty liver disease in children and adolescence: from "two hit theory" to "multiple hit model". World J Gastroenterol. 2018;24:2974-83.

16. Athyros VG, Tziomalos K, Daskalopoulos GN, Karagiannis A, Mikhailidis DP. Statin-based treatment for cardiovascular risk and non-alcoholic fatty liver disease. Killing two birds with one stone. Ann Med. 2011;43:167-71.

17. Chatrath $H$, Vuppalanchi $R$, Chalasani N. Dyslipidemia in patients with nonalcoholic fatty liver disease. Semin Liver Dis. 2012;32:22-9.

18. Musso G, Gambino R, Cassader M. Cholesterol metabolism and the pathogenesis of non-alcoholic steatohepatitis. Prog Lipid Res. 2013;52:17591.

19. Polyzos SA, Aronis KN, Kountouras J, Raptis DD, Vasiloglou MF, Mantzoros CS. Circulating leptin in non-alcoholic fatty liver disease: a systematic review and meta-analysis. Diabetologia. 2016:59:30-43.

20. Reddy JK, Rao MS. Lipid metabolism and liver inflammation. II. Fatty liver disease and fatty acid oxidation. Am. J. Physiol. Gastrointest. Liver Physiol. 2006;290:G852-8.

21. Ferramosca A, Zara V. Modulation of hepatic steatosis by dietary fatty acids. World J Gastroenterol. 2014;20:1746-55.

22. Mashek DG, Khan SA, Sathyanarayan A, Ploeger JM, Franklin MP. Hepatic lipid droplet biology: getting to the root of fatty liver. Hepatology. 2015;62: 964-7.

23. Expert Panel on Detection, Evaluation, Adults aToHBCi. Executive summary of the third report of the National Cholesterol Education Program (NCEP) expert panel on detection, evaluation, and treatment of high blood cholesterol in adults (adult treatment panel III). JAMA. 2001;285:2486-97.

24. Wang D, Wang L, Wang Z, Chen S, Ni Y, Jiang D. Higher non-HDLcholesterol to HDL-cholesterol ratio linked with increased nonalcoholic steatohepatitis. Lipids Health Dis. 2018;17:67.

25. Brunt EM. Pathology of nonalcoholic fatty liver disease. Nat Rev Gastroenterol Hepatol. 2010;7:195-203.

26. Welsh JA, Karpen S, Vos MB. Increasing prevalence of nonalcoholic fatty liver disease among United States adolescents, 1988-1994 to 2007-2010. J Pediatr. 2013;162:496-500.e1.

27. Liu Z, Que S, Zhou L, Zheng S. Dose-response relationship of serum uric acid with metabolic syndrome and non-alcoholic fatty liver disease incidence: a meta-analysis of prospective studies. Sci Rep. 2015;5:14325.

28. Ballestri S, Nascimbeni F, Baldelli E, Marrazzo A, Romagnoli D, Lonardo A. NAFLD as a sexual dimorphic disease: role of gender and reproductive status in the development and progression of nonalcoholic fatty liver disease and inherent cardiovascular risk. Adv Ther. 2017;34:1291-326.

29. Saadeh S, Younossi ZM, Remer EM, Gramlich T, Ong JP, Hurley M, et al. The utility of radiological imaging in nonalcoholic fatty liver disease. Gastroenterology. 2002;123:745-50.

\section{Publisher's Note}

Springer Nature remains neutral with regard to jurisdictional claims in published maps and institutional affiliations. 